REVIEW

\title{
Transition from child- to adult-orientated care for children with long-term health conditions: A process, not an event
}

\author{
A Westwood, ${ }^{1}$ MD, FCP (SA); N Langerak, ${ }^{2}$ BSc (PT) (Utrecht), MSc (Nijmegen), PhD; G Fieggen, ${ }^{2}$ MSc (Lond), MD, FCS (SA) \\ ${ }^{1}$ School of Child and Adolescent Health, Faculty of Health Sciences, University of Cape Town, and General Paediatrics, Metro West, \\ Department of Health, Provincial Government of the Western Cape, Cape Town, South Africa \\ ${ }^{2}$ Division of Neurosurgery, Faculty of Health Sciences, University of Cape Town, South Africa
}

Corresponding author: A Westwood (anthony.westwood@westerncape.gov.za)

This month's CME component contains the second of a two-part series of continuing medical education articles on various aspects of spina bifida, with the focus on some of the longer-term management issues such as hydrocephalus and limb deformity, as well as quality of life.

S Afr Med J 2014;104(4):310-313. DOI:10.7196/SAMJ.8201

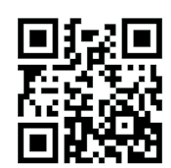

Spina bifida is an example of a 'long-term health condition', a term preferred to 'chronic disease' (the standard term for adult continuing health problems) in the paediatric lexicon for a number of reasons. Given the child's continuous development, time spent living with a health condition can lead to secondary health problems or disabilities in spheres such as growth and psychosocial development. The term 'health condition' is preferred as many of the problems that afflict children and require medical support are not diseases. Spina bifida is a prime illustration of this important distinction - the need for long-term use of health services as described in the accompanying articles, but not being primarily a disease. Importantly, in this context, children with disabilities are not dichotomised from those with diseases; they have similar needs in terms of health services and face similar challenges to their development and in their negotiation of life's transitions.

With improved survival of children with long-term health conditions, there is a need for medical care into adulthood and attention needs to be paid to the process by which the transition from child- to adult-centred care is made. This is of relevance to the primary care practitioner who can play a crucial role in ensuring the success of this process.

\section{Long-term health conditions}

A long-term health condition has been defined by Stein et al. ${ }^{[1]}$ as a disorder that:
- has a biological, psychological cognitive basis

- has lasted or is virtually certain to last at least a year

- produces one or more of the following sequelae:

- limitation of function

- dependency on medications, special diet, medical technology, assistive device, or personal assistance

- needs medical care or related services, psychological services and educational services over and above the usual for the child's age.

This so-called non-categorical approach promotes a generic process towards the care of children with long-term health conditions, emphasising commonalities such as psychological stresses, the need for planning of emergency care, and negotiating adolescence.

Therefore, care of children with long-term health conditions needs to be tailored to the individual condition and to the many implications of the presence of the condition over time in domains such as education, psychosocial development, linear growth, family functioning, and negotiation of life stages. The more complex the condition, the more complicated the care becomes, and concomitantly the more the risks of secondary disability and maladjustment rise. With the recent advances in medical and surgical care, many children with previously fatal health problems are surviving to and through adolescence into adulthood - with complex disorders. This demographic change brings with it new challenges. In particular, the so-called 'tasks' of adolescence - namely 'spousing' (finding a life partner), 'carousing' (making lifestyle decisions), 'housing' 
(developing independence and autonomy) and 'job market browsing' (finding a career) - are rendered more challenging and risky in the presence of a complex long-term health condition.

For many children with milder conditions such as atopic diseases, care through childhood and adolescence into adulthood may occur within the primary healthcare context, with little turbulence as the life stages pass.

However, most children and adolescents with more complex long-term health conditions require specialised healthcare, often rendered by a multidisciplinary team. Unfortunately the primary care practitioner is often not included in this team.

\section{Transition of care}

Adolescents growing older with a complex long-term health condition (and their families) almost inevitably face a change of healthcare provider/s. Specialised healthcare has to a large extent dichotomised children and adults, with adolescents often left out in the cold. This situation presents many challenges to those dealing with complex long-term health conditions. In particular, negotiating the 'transition' from paediatric care through adolescent-orientated care to adult care requires attention. This transition has been the subject of much discussion and many descriptions, but there has been limited research into outcomes of the transition processes. This article attempts to provide insights into this literature.

Twenty years ago the Society of Adolescent Medicine defined transition as the purposeful planned movement of adolescents and young adults with chronic physical and medical conditions from child-centred to adult-centred healthcare systems. ${ }^{[2]}$ It was acknowledged then that much was unknown about the process. The need for the transition process to be studied and evaluated was emphasised. Ten years later the same society lamented: 'Although transition principles have been described and a variety of models have been proposed and implemented, outcome data are still lacking, especially those that would support one model over another. ${ }^{[3]}$

Over the last 20 years, many models have been described, theories expounded and opinions given, but there is little research to guide healthcare providers. This is not surprising, given the complex and varying situations in which transition has to occur. Health services differ, health conditions do not have the same implications and vary in severity, and significant numbers of patients and families followed up over lengthy periods are required to provide valid conclusions in any interventional research.

\section{What is known about the process of transition and what impedes or promotes it}

In an article analysing transition in cystic fibrosis, Schidlow and Feil ${ }^{[4]}$ usefully spelt out generic factors related to the patient, the family and the healthcare providers that enhance or hinder a successful transition (Table 1). In the South African context, 'economic concerns' might not be expected to have the prominence given in this American perspective. Rosen ${ }^{[5]}$ pointed out the cultural differences between child- and adultorientated services that may impede successful transition: in effect, the paediatric environment is nurturing while the adult one expects independent behaviour from the patient. In the long-term health condition arena, the former could inhibit the patient's maturation, while the latter expects too much maturity. A child with a long-term health condition needs to be encouraged to take on the health problem and its implications, while the parents need to be encouraged to step back; the adult healthcare provider must not expect a fully fledged adult even if the patient is of adult age. The patient's family usually still has a significant role to play in the life of the adult with a long-term health condition.

Table 1. Factors that influence the success of transition from child- to adult-orientated healthcare ${ }^{[4]}$

\begin{tabular}{|c|c|c|c|}
\hline \multirow[t]{7}{*}{ The patient } & Dependent behaviour & The paediatric & Economic concerns about programme \\
\hline & Immaturity & caregivers & Emotional bonds with patient and family \\
\hline & Severe illness or disability & & Comfort with status quo \\
\hline & Psychopathology & & Perception of own skills as caregivers of adults \\
\hline & Lack of support systems & & Perception of potential survival of patients \\
\hline & Lack of trust in caregivers & & Distrust of adult caregivers \\
\hline & Poor adherence to treatment regimens & & Ambivalence towards transition and transfer of care \\
\hline \multirow[t]{7}{*}{ The family } & Excessive need for control & The adult caregivers & Economic concerns \\
\hline & Emotional dependency & & Lack of understanding of congenital disease \\
\hline & Psychopathology & & Lack of familiarity with disease entities \\
\hline & Parenting styles leading to overprotection & & Heightened perception of care demands \\
\hline & Heightened perception of disease severity & & Lack of institutional commitment \\
\hline & Lack of trust in caregivers & & \\
\hline & Mistaken perception of potential survival & & \\
\hline
\end{tabular}


For children with significant neurodisability and cognitive difficulties, this transition is made even more complex. In an unpublished study undertaken in Cape Town in the 1990s, we found that the greatest difficulty in transition was in the neurology clinic, while the simplest transition took place in the neurosurgical service. The former has a high incidence of secondary epilepsy together with motor and cognitive difficulty, which the adult neurology service felt was not their core business and yet was (and still is) not covered well anywhere else in the health system; the neurosurgical division had staff who covered both the adult and child services (Westwood A, Henley L - unpublished report 1998). These case studies illustrate how individual health conditions and health services can impede or encourage successful transition to adult services.

The biggest impediment to transition is the shortage of suitable adult services due to a lack of expertise in childhood-onset health conditions among adult service providers. This has become less of an impediment in many countries as numbers of young people growing up with conditions such as congenital heart disease and cystic fibrosis have grown. How well prepared is an adult endocrinologist or physician in South Africa to deal with the young person who has had type 1 diabetes mellitus for years? South Africa faces a particular challenge with regard to its expanding and ageing paediatric HIVinfected population. The issues have been well described in a technical brief by USAID and PEPFAR in 2012. ${ }^{[6]}$

Importantly, age-based criteria for the transfer to the adult service are to be avoided. Given the wide range of levels of maturity and readiness that are likely to be found among young people, such rigid criteria are unlikely to promote successful transition. In a study of patient and parent attitudes towards transition before and after the process in the cystic fibrosis population, age-related criteria were not supported. ${ }^{[7]}$ They also do not make sense developmentally. This approach has been strongly supported by the Society for Adolescent Medicine ${ }^{[3]}$ and the American Academy of Pediatrics. ${ }^{[8]}$

\section{What transition models exist and which are likely to work?}

Given the complexity of transition and the lack of a large research base, there are many potentially valid models of transition. An important theme would seem to be exposure of the young person and family to the adult care providers. This can be achieved through visits to the paediatric and adult services in the years before formal transfer. Where patient numbers are adequate, adolescent transition clinics (shared by the paediatric and adult teams) have been successful. Among other advantages, these clinics provide peer support that facilitates safe passage to the adult service.

The recent literature has explored the concept of a transition co-ordinator, first proposed in the position paper from the Society for Adolescent Medicine. ${ }^{[2]}$ This health team member is envisaged as spearheading preparations for patients and families, while also serving as a conduit between old and new team members. This co-ordinator will usually be a nurse, but could be a primary care practitioner.

Recently, Schwartz et al. ${ }^{[9]}$ attempted to operationalise the model of transition readiness that takes account of the phases of care as well as the individual factors of 'socio-ecology' in the child, parent and providers. In this model multiple assessments are done before the actual transfer takes place.

The model espoused for HIV transition care in Africa is that of family-centred care 'delivered by a multidisciplinary team that provides support for adolescents with HIV infection, with the adolescent infected perinatally or by sexual transmission, as well as the parent or guardian caregivers ... ${ }^{\left[{ }^{[10]}\right.}$ This approach reflects the special needs of HIV care in the African context where HIV tends to be a family disease, and is a unique model in the transition literature.

This is the closest model one finds to a primary care-based model for transition and it challenges the assumption that co-ordination of care, including transition, needs to be led by specialised services. However, in South Africa there is currently very limited primary healthcare engagement with children with more complex long-term health conditions, especially during adolescence. This does not preclude exploration of the role of primary healthcare in transition for young people with long-term health conditions, and perhaps HIV, now the commonest complex long-term health condition in this country, will lead the way.

\section{Specific issues in spina bifida}

The value of a multidisciplinary clinic is well established, although such clinics appear to be more common in public teaching hospitals than in private practice. It also seems easier to organise such clinics in a paediatric setting than an adult service where care tends to fragment into individual specialties. Various surgical disciplines such as urology and neurosurgery need to follow up these patients through adulthood, as the life-threatening consequences of the neuropathic bladder or shunt obstruction are well known. Loss of ambulation in late childhood is a well-recognised problem ${ }^{[11,12]}$ that may be due to accepting the improved energy efficiency of a wheelchair, psychological factors or weight gain, but subtle neurological deterioration due to shunt dysfunction or a tethered spinal cord must be excluded. ${ }^{[13]}$ Consideration must also be given to sexuality and planning for pregnancy and delivery.

\section{Transition in cerebral palsy}

It is worth emphasising that people with other conditions associated with neurodisability, such as cerebral palsy, also face significant challenges in transition of care. This was clearly apparent to the authors in a long-term follow-up study of young adults who had undergone the neurosurgical procedure of selective dorsal rhizotomy in Cape Town. Twenty years after surgery, all the patients continued to experience sustained improvement in spasticity but none was 
receiving physiotherapy or any regular medical follow-up, despite persistence of the underlying condition. ${ }^{[14]}$

A recent review of functional levels in adolescents and children with cerebral palsy pointed to the need for specific co-ordinated care, including preventive care, access to emergency services and involvement of subspecialists, which should start early in the teenage years. ${ }^{[15]}$ Information systems that provide specific care protocols are of value, as well as a registry that can be used for tracking physical, behavioural and functional health status. Ensuring adequate medical care should be coupled with encouraging independence, particularly with regard to financial management and access to community resources. Modern tools, such as an online support systems, may help mentor people during their transition and thereafter connect them with a cerebral palsy community, making them less isolated and strengthening their companionship and independence.

\section{Conclusion}

Transition must be prepared for and planned during childhood from the time of diagnosis of the long-term health condition. The family and child require support to promote the young person's ability to take ownership of his or her health situation and therapies by the time of adolescence. The paediatric and primary healthcare teams bear responsibility for this. Bilateral systems that bridge and break down the divide between child- and adult-orientated care must be set up. Many mechanisms exist that can be adapted to specific health conditions and health service configurations. Adult-orientated service needs to ensure that there is adequate understanding of the formerly paediatric-only conditions and of young adults and their families so that the 'medical home' of paediatrics and primary care can also be found in adult services.

\section{Summary}

- Long-term health conditions in childhood include both congenital conditions and acquired diseases.

- Children with long-term health conditions face issues and potential secondary problems that are different from those of adults with chronic diseases.
- Transition to adult-orientated care for such children and adolescents is a major challenge.

- Transition needs to be prepared for and planned.

- A variety of possible transition models exists, depending on circumstances.

\section{References}

1. Stein REK, Bauman LJ, Westbrook LE, Coupey SM, Ireys HT. Framework for identifying children who have chronic conditions: The case for a new definition. J Pediatr 1993;122:342-347. [http:// dx.doi.org/10.1016/S0022-3476(05)83414-6]

2. Blum RWM, Garell D, Hodgman CH, et al. Transition from child-centered to adult health-care systems for adolescents with chronic conditions. J Adolesc Health 1993;14:570-576. [http://dx.doi. org/10.1016/1054-139X(93)90143-D]

3. Rosen DS, Blum RW, Britto M, Sawyer SM, Siegel DM. Transition to adult health care for adolescents and young adults with chronic conditions: Position paper of the Society for Adolescent Medicine. J Adolesc Health 2003;33:309-311.

4. Schidlow DV, Feil SB. Life beyond pediatrics. Med Clin North Am 1990;74:1113-1120.

5. Rosen D. Between two worlds: Bridging the cultures of child health and adult medicine. J Adolesc Health 1995;17:10-16. [http://dx.doi.org/10.1016/1054-139X(95)00077-6]

6. Sharer M, Fullem A. Transitioning of Care and Other Services for Adolescents Living With HIV in Sub-Saharan Africa: Technical Brief. USAID and PEPFAR. Arlington, VA: AIDSTAR-One, 2012.

7. Westwood ATR, Henley LD, Willcox P. The transition from paediatric to adult care for persons with Westwood ATR, Henley LD, Willcox P. The transition from paediatric to adult care for persons with
cystic fibrosis: Patient and parent perspectives. J Paediatr Child Health 1999;35:442-445. [http:// cystic fibrosis: Patient and parent perspectives.
dx.doi.org/10.1046/j.1440-1754.1999.355394.x]

8. American Academy of Pediatrics, American Academy of Family Physicians, American College of Physicians-American Society of Internal Medicine. Consensus statement on health care transitions for young adults with special health care needs. Pediatrics 2002;110:1304-1306.

9. Schwartz LA, Tuchmann LK, Hobbie WL, Ginsberg JP. A social-ecological model of readiness from transition to adult-oriented care for adolescents and young adults with chronic health conditions. Child Care Health Dev 2011;37:883-895.

10. Coovadia H, Mantell JE. Adolescents and HIV in sub-Saharan Africa: A timely issue and missed Coovadia H, Mantell JE. Adolescents and HIV in sub-Saharan Africa: A timely
opportunity. Clin Infect Dis 2011;51:852-854. [http://dx.doi.org/10.1086/656362]

1. Bowman RM, McLone DG, Grant JA, Tomita T, Ito JA. Spina bifida outcome: A 25-year prospective. Pediatr Neurosurg 2001;34:114-120.

2. Vinchon M, Dhellemmes P. The transition from child to adult in neurosurgery. Adv Tech Stand Neurosurg 2007;32:3-24.

13. Binks I, Barden JS, Burke TA, Young NL. What do we really know about the transition to adultBinks I, Barden SS, Burke TA, Young NL. What do we really know about the transition to adult-
centered health care? A focus on cerebral palsy and spina bifida. Arch Physical Med Rehab centered health cas

14. Langerak NG, Hillier SL, Verkoeijen PP, Peter JC, Fieggen AG, Vaughan CL. Level of activity and Langerak NG, Hillier SL, Verkoeijen PP, Peter JC, Fieggen AG, Vaughan CL. Level of activity and
participation in adults with spastic diplegia 17-26 years after selective dorsal rhizotomy. J Rehabil participation in adults with spastic diplegia 17-26 years after selectiv

15. Frisch DF, Msall ME. Health, functioning, and participation of adolescents and adults with cerebral palsy: A review of outcomes research 2013;18:84-94.

\section{Further reading}

- Sawyer SM, Drew S, Yeo M, Britto M. Adolescents with a chronic condition: Challenges living, challenges treating. Lancet 2009;369:1481-1489.

Watson R, Parr JR, Joyce C, May C, Le Couteur AS. Models of transitional care for young people with complex health needs: A scoping review. Child Care Health Dev 2011;37:780-791 